\title{
Microscopic formulation of the interacting boson model for rotational nuclei
}

\author{
Kosuke Nomura, ${ }^{1}$ Takaharu Otsuka,,${ }^{1,2,3}$ Noritaka Shimizu, ${ }^{1}$ and Lu Guo ${ }^{4}$ \\ ${ }^{1}$ Department of Physics, University of Tokyo, Hongo, Bunkyo-ku, Tokyo 113-0033, Japan \\ ${ }^{2}$ Center for Nuclear Study, University of Tokyo, Hongo, Bunkyo-ku, Tokyo 113-0033, Japan \\ ${ }^{3}$ National Superconducting Cyclotron Laboratory, Michigan State University, East Lansing, Michigan 48824, USA \\ ${ }^{4}$ RIKEN Nishina Center, Hirosawa, Wako-shi, Saitama 351-0198, Japan \\ (Received 5 March 2011; published 25 April 2011; publisher error corrected 2 May 2011)
}

\begin{abstract}
We propose a novel formulation of the interacting boson model (IBM) for rotational nuclei with axially symmetric, strong deformation. The intrinsic structure represented by the potential-energy surface (PES) of a given multinucleon system has a certain similarity to that of the corresponding multiboson system. Based on this feature, one can derive an appropriate boson Hamiltonian, as already reported. This prescription, however, has a major difficulty in the rotational spectra of strongly deformed nuclei: the bosonic moment of inertia is significantly smaller than the corresponding nucleonic one. We present that this difficulty originates in the difference between the rotational response of a nucleon system and that of the corresponding boson system, and could arise even if the PESs of the two systems were identical. We further suggest that the problem can be solved by implementing the $\hat{L} \cdot \hat{L}$ term into the IBM Hamiltonian, with the coupling constant derived from the cranking approach of Skyrme mean-field models. The validity of the method is confirmed for rare-earth and actinoid nuclei, as their experimental rotational yrast bands are reproduced nicely.
\end{abstract}

DOI: 10.1103/PhysRevC.83.041302

PACS number(s): 21.10.Re, 21.60.Ev, 21.60.Fw, 21.60.Jz

The atomic nucleus is a strongly interacting, many-body quantal system which has collective properties resulting in various deformed shapes. If a nucleus is strongly deformed, it rotates, exhibiting the characteristic rotational-band structure with remarkable regularity. Such rotational motion can be viewed as a manifestation of the symmetry-restoration mechanism of Nambu, and has attracted much attention in nuclear physics from various viewpoints $[1,2]$.

The interacting boson model (IBM) $[3,4]$ has been successful in phenomenological studies for describing low-lying, quadrupole collective states of medium-heavy nuclei [3]. The major assumption of the IBM is to employ $L=0^{+}(s)$ and $L=2^{+}(d)$ bosons, which reflect the collective $S$ and $D$ pairs of valence nucleons [5]. The microscopic foundation of the IBM has been studied extensively so as to derive an IBM Hamiltonian starting from nucleon degrees of freedom [5-7]. An alternative approach to derive the IBM Hamiltonian has been presented recently [8]. In this approach, the potentialenergy surface (PES) with quadrupole degrees of freedom, obtained from the mean-field calculation with the Skyrme energy density functional (EDF) $[9,10]$, is compared to the corresponding PES of the IBM to obtain the parameters of the IBM Hamiltonian. As a Skyrme EDF gives universal descriptions of various nuclear properties [2,9-11], one can derive the IBM Hamiltonian basically for all situations in a unified way. This method is valid particularly for nuclei with weak to moderate quadrupole deformation, and has been practiced extensively [12]. When a nucleus is well deformed, however, the nucleonic rotational spectrum appears notably and systematically different from the corresponding bosonic one, being manifested by a too-small bosonic moment of inertia as compared to the corresponding fermionic one $[8,12]$.

This kind of difference has been known to be a result of limited degrees of freedom, with $s$ and $d$ bosons only, in many cases $[13,14]$. In order to remedy this problem, another type of nucleon pairs, e.g., $L=4^{+}(G)$ pair, and the corresponding boson image ( $g$ boson) have been introduced, and their effects have been renormalized into a $s d$-boson sector, yielding IBM Hamiltonians consistent with phenomenological ones [6,7,13-21]. In the meantime, the validity of the IBM for rotational nuclei was analyzed in terms of the Nilsson model [22], resulting in the criticism that the $S D$-pair truncation may be far from sufficient to describe the intrinsic states of strongly deformed nuclei, and this naturally casts a question on the applicability of the IBM to rotational nuclei in particular. While it has been reported that the $S D$-pair dominance holds to a good extent in intrinsic states of rotational nuclei $[15,23,24]$, there has been no conclusive mapping procedure from nucleonic systems to IBM ones covering rotational nuclei. It is thus of much interest to revisit this issue with the proposed method of Ref. [8], looking for a prescription to cure the aforementioned problem of a too-small moment of inertia.

In the method of Ref. [8], we calculate the energies of nucleonic and bosonic intrinsic states representing various shapes, and obtain PESs. We then determine the parameters of the IBM Hamiltonian so that the bosonic PES becomes similar to the nucleonic one [8]. These intrinsic states are at rest with rotational frequency $\omega=0$. In this Rapid Communication, we move one step further with nonzero rotational frequency $\omega \neq 0$. Actually we analyze the responses of the nucleonic and bosonic intrinsic states by rotational cranking with infinitesimal $\omega$. From such responses, one can extract the most important rotational correction to the IBM Hamiltonian.

The nucleon intrinsic state $\left|\phi_{F}\right\rangle$ is obtained from the Hartree-Fock plus BCS (HF + BCS) calculation. The Skyrme $\mathrm{SkM}^{*}$ interaction [26] is used throughout, while different Skyrme forces do not alter the conclusion. 
For the boson system, we consider the IBM-2 because it is closer to a microscopic picture than the simpler version of the IBM. The IBM-2 is comprised of proton $L=0^{+}\left(s_{\pi}\right)$ and $2^{+}\left(d_{\pi}\right)$ bosons, and of neutron $L=0^{+}\left(s_{v}\right)$ and $2^{+}\left(d_{v}\right)$ bosons [5]. We take the standard IBM-2 Hamiltonian

$$
H_{B}=\epsilon n_{d}+\kappa Q_{\pi} \cdot Q_{\nu},
$$

where $n_{d}=n_{d \pi}+n_{d v}$, with $n_{d \pi}\left(n_{d v}\right)$ being the proton (neutron) $d$-boson number operator and $Q_{\rho}=s_{\rho}^{\dagger} \tilde{d}_{\rho}+d_{\rho}^{\dagger} \tilde{s}_{\rho}+$ $\chi_{\rho}\left[d_{\rho}^{\dagger} \tilde{d}_{\rho}\right]^{(2)}$. Here, $\epsilon, \kappa$, and $\chi_{\pi, v}$ are parameters, and their values are determined by comparing nucleonic and bosonic PESs following Refs. [8,12]. The boson intrinsic state $\left|\phi_{B}\right\rangle$ is written in general as a coherent state [27,28]

$$
\left|\phi_{B}\right\rangle \propto \prod_{\rho=\pi, \nu}\left(s_{\rho}^{\dagger}+\sum_{\mu= \pm 2, \pm 1,0} a_{\rho \mu} d_{\rho \mu}^{\dagger}\right)^{n_{\rho}}|0\rangle,
$$

where $|0\rangle$ and $a_{\rho \mu}$ represent the boson vacuum (inert core) and amplitude, respectively.

We now look into the problem of rotational response. We shall restrict ourselves to nuclei with axially symmetric, strong deformation, because this problem is crucial to those nuclei but is not so relevant to the others. An axially symmetric intrinsic state is invariant with respect to the rotation around the symmetry ( $z$ ) axis. This means $a_{\rho \mu}=0$ for $\mu \neq 0$ in Eq. (2) in the case of bosons. Such intrinsic states of nucleons and bosons are supposed to be obtained as the minima of the PESs. Let us now rotate the axially symmetric intrinsic states about the $y$ axis by angle $\beta$. Figure 1 shows the overlap between the intrinsic state $\left|\phi_{X}\right\rangle$ and the rotated one $\left|\phi_{X}^{\prime}\right\rangle=e^{-i L_{y} \beta}\left|\phi_{X}\right\rangle$, where $X$ stands for either fermion $(X=F)$ or boson $(X=B)$. Here, $L_{y}$ denote the $y$ component of the angular momentum operator. We take ${ }^{146-154} \mathrm{Sm}$ and ${ }^{230-238} \mathrm{U}$ as examples. Some of these nuclei are good examples of the SU(3) limit of the IBM [25].

Figures 1(a)-1(d) show the overlaps for nucleons and bosons, respectively. For $\mathrm{Sm}$ isotopes, the parameters of $H_{B}$ are taken from [12], while the parameters for $\mathrm{U}$ isotopes are determined as $\epsilon \approx 0.100 \mathrm{MeV}, \kappa \approx-0.18 \mathrm{MeV}$, and $\chi_{\pi} \approx \chi_{\nu} \approx-1.0$. These parameters are used throughout this Rapid Communication.

In each case, the overlap is peaked at $\beta=0^{\circ}$ with the value unity, and decreases with $\beta$. The nucleonic overlaps are peaked more sharply, whereas boson ones are damped more slowly. It is clear that as a function of $\beta$, the boson rotated intrinsic state changes more slowly than the corresponding nucleon one, due to limited degrees of freedom for bosons.

We point out that the overlap becomes narrower in $\beta$ with the neutron number $N$ for Sm isotopes [see Figs. 1(a) and 1(b)]. This is related to the growth of deformation. However, there is no notable change in the overlap for these $U$ isotopes, because a pronounced prolate minimum appears always at $\beta_{2} \sim 0.25$ in their PES.

The nucleon-boson difference of the rotational response discussed so far suggests that the rotational spectrum of a nucleonic system may not be fully reproduced by the boson system determined by the mapping method of Ref. [8] using the PESs at rest. In fact, it will be shown later that the
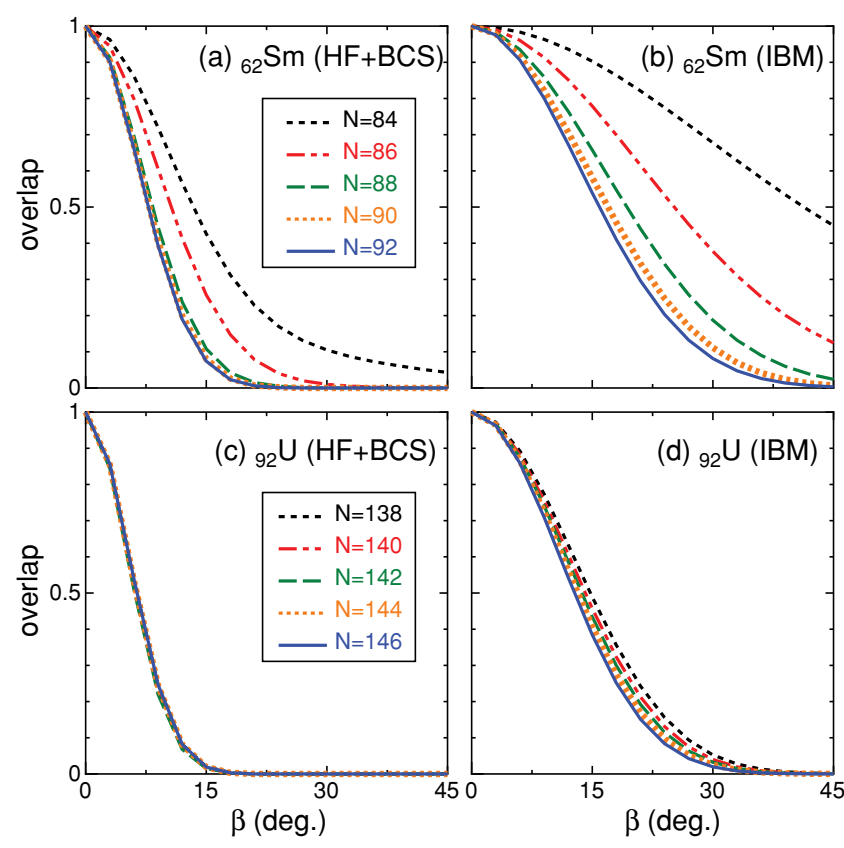

FIG. 1. (Color online) Overlap between the intrinsic state and its rotation at angle $\beta$ for ${ }^{146-154} \mathrm{Sm}$ and ${ }^{230-238} \mathrm{U}$ nuclei for (a), (c) fermion (HF + BCS) and (b), (d) boson (IBM) systems.

moment of inertia of a nucleon system differs from the one calculated by the mapped boson Hamiltonian. We then introduce a term into the boson Hamiltonian, so as to keep the PES-based mapping procedure, but incorporate the different rotational responses. This term takes the form of $\hat{L} \cdot \hat{L}$, where $\hat{L}$ denotes the boson angular momentum operator. This term is nothing but the squared magnitude of the angular momentum with the eigenvalue $L(L+1)$, and changes the moment of inertia of the rotational band, keeping their wave functions. A phenomenological term of this form was used in the fitting calculation of the IBM, particularly in its SU(3) limit [25], without knowing its origin or physical significance.

We adopt, hereafter, a Hamiltonian $H_{B}^{\prime}$ which includes this term with coupling constant $\alpha$,

$$
H_{B}^{\prime}=H_{B}+\alpha \hat{L} \cdot \hat{L}
$$

where $H_{B}$ is given in Eq. (1). The $\alpha \hat{L} \cdot \hat{L}$ term will be referred to as the LL term hereafter. The LL term contributes to the PES in the same way as a change of $d$-boson energy $\Delta \epsilon=6 \alpha$ [see Eq. (1)], because the PES at rest (i.e., $\omega=0$ ) is formed by the boson intrinsic state $\left|\phi_{B}\right\rangle$ containing no $d_{ \pm 1}$ component. Hence, by shifting $\epsilon$ slightly, we obtain the same PES as the one without the LL term, and consequently the other parameters of mapped $H_{B}$ remain unchanged.

We now turn to the determination of $\alpha$ in Eq. (3). First, we perform the cranking model calculation for the fermion system to obtain its moment of inertia, denoted by $\mathcal{J}_{F}$, in the usual way [2]. By taking the Inglis-Belyaev (IB) formula, we obtain $[29,30]$

$$
\mathcal{J}_{F}=2 \sum_{i, j>0} \frac{\left|\left\langle i\left|L_{k}\right| j\right\rangle\right|^{2}}{E_{i}+E_{j}}\left(u_{i} v_{j}-u_{j} v_{i}\right)^{2},
$$


where energy $E_{i}$ and $v$ factor $v_{i}$ of the quasiparticle state $i$ are calculated by the HF + BCS method of Ref. [31]. Here, $L_{k}$ is the nucleon angular momentum operator, and $k$ means the axis of the cranking rotation, being either $x$ or $y$, as $z$ axis. Based on the earlier argument, the $y$ axis is chosen.

Next, the bosonic moment of inertia, denoted as $\mathcal{J}_{B}$, is calculated by the cranking formula of Ref. [32] with $d_{ \pm 1}$ being mixed, to an infinitesimal order, into the coherent state $\left|\phi_{B}\right\rangle$ in Eq. (2),

$$
\mathcal{J}_{B}=\lim _{\omega \rightarrow 0} \frac{1}{\omega} \frac{\left\langle\phi_{B}\left|L_{k}\right| \phi_{B}\right\rangle}{\left\langle\phi_{B} \mid \phi_{B}\right\rangle},
$$

where $\omega$ is the cranking frequency, $a_{\rho \pm 1}$ denotes the amplitude for $d_{\rho \pm 1}$, and $L_{k}$ stands for the boson angular momentum operator. Note that $a_{\rho \pm 1} \propto \omega$ at this limit, leading $\mathcal{J}_{B}$ to a finite value.

The value of $\alpha$ is determined for the individual nucleus so that the corresponding bosonic moment of inertia $\mathcal{J}_{B}$ in Eq. (5) becomes equal to $\mathcal{J}_{F}$ in Eq. (4). This prescription makes sense, if the nucleus is strongly deformed and the fixed intrinsic state is so stable as to produce individual levels of a rotational band through the angular momentum projection in a good approximation. The resultant excitation energies should follow the rotor formula $E_{x} \propto L(L+1)$ for $L$ being the angular momentum of the level. The present prescription with the LL term should be applied only to certain nuclei which belong to this type. We introduce a criterion to select such nuclei in terms of the ratio $R_{4 / 2}=E_{x}\left(4_{1}^{+}\right) / E_{x}\left(2_{1}^{+}\right)$, and set a minimum value for this. Empirical systematics [33] suggest that the evolution toward stronger deformation continues as the number of valence nucleons increases, but this evolution becomes saturated beyond $R_{4 / 2} \sim 3.2$. Namely, for the nuclei with $R_{4 / 2}>3.2$, the deformation is considered to be evolved sufficiently well, and we take $R_{4 / 2}>3.2$ as the criterion to apply the LL term. This discrete criterion is also for the sake of simplicity, but the major discussions of this work do not depend on its details.

Figures 2(a)-2(c) show the moments of inertia for Sm and $\mathrm{U}$ isotopes. In these figures, $\mathcal{J}_{B}$ calculated with the LL term (w/LL), $\mathcal{J}_{B}$ calculated without it (w/o LL), and $\mathcal{J}_{F}$ are compared. Experimental ones determined from the $2_{1}^{+}$ levels [35] are shown also.

We divide $\mathrm{Sm}$ isotopes into two categories according to the criterion defined above. First, the ratio $R_{4 / 2}$ is calculated without the LL term, leading to ${ }^{152-158} \mathrm{Sm}$ with $R_{4 / 2}>3.2$ and ${ }^{146-150} \mathrm{Sm}$ with $R_{4 / 2}<3.2$. For the former category, the LL term should be included, and Fig. 2(b) demonstrates that the LL term produces significant effects so as to be just enough for the agreement to the experiment. To be more precise, the experimental value becomes large for $N=90$, and looks nearly flat for $N \geqslant 92$, being $35-40 \mathrm{MeV}^{-1}$. The enlargement of the moment of inertia means that the value of $\alpha$ is negative. The IB formula reproduces this trend quite well, which is applied to bosons by the present method.

Although the LL term should not be used for the category depicted in Fig. 2(a), we shall study some features. The increase of $\mathcal{J}_{F}$ and $\mathcal{J}_{B}$ with increasing $N$ can be seen. Although the experimental moment of inertia exhibits a gap between Figs. 2(a) and 2(b) (from $N=88$ to 90), neither

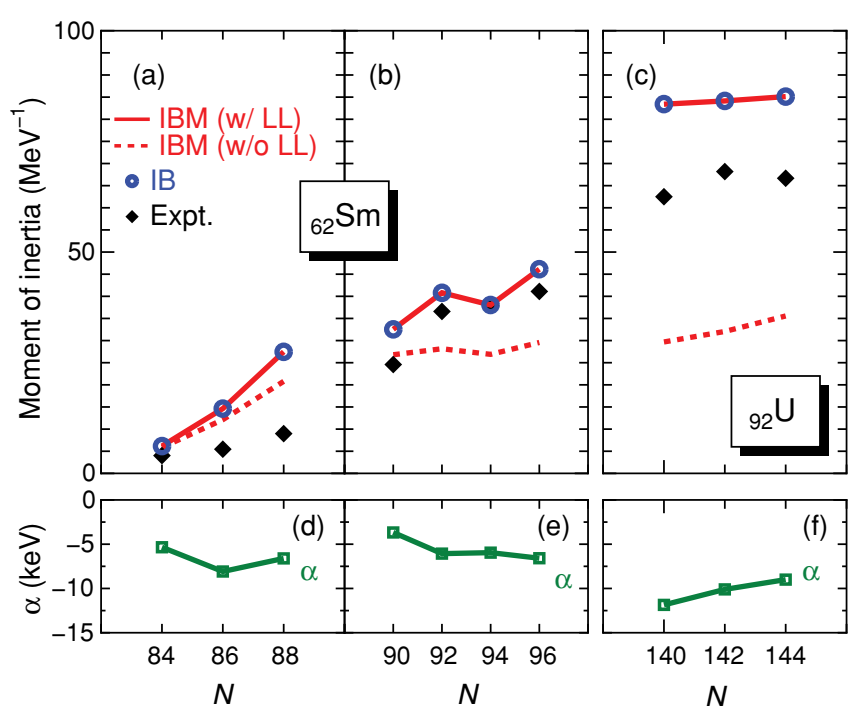

FIG. 2. (Color online) Upper panels: Moments of inertia in the intrinsic state for (a) ${ }^{146-150} \mathrm{Sm}$, (b) ${ }^{152-158} \mathrm{Sm}$, and (c) ${ }^{232-236} \mathrm{U}$, calculated using the IBM with (w/) and without (w/o) the LL term and by the IB formula. Experimental data taken from $E_{x}\left(2_{1}^{+}\right)$[35] are also shown. Lower panels: The derived $\alpha$ value for (d) ${ }^{146-150} \mathrm{Sm}$, (e) ${ }^{152-158} \mathrm{Sm}$, and (f) ${ }^{232-236} \mathrm{U}$.

$\mathcal{J}_{B}$ nor $\mathcal{J}_{F}$ follow this trend, showing only gradual changes. This could be due to the absence of the particle-number conservation in the Skyrme EDF calculation. We do not touch on this point in this Rapid Communication.

Figures 2(d) and 2(e) show, respectively, the derived $\alpha$ value for ${ }^{146-150} \mathrm{Sm}$ and ${ }^{152-158} \mathrm{Sm}$. First we notice an overall trend that $\alpha$ does not change very much, while the IB value of $\mathcal{J}_{F}$ changes by an order of magnitude. Although the $\alpha$ values for ${ }^{146-150} \mathrm{Sm}$ do not make much sense, this is of certain interest.

Figure 2(c) shows the moments of inertia for ${ }^{232-236} \mathrm{U}$, which are rather flat. We point out that the calculated moment of inertia $\mathcal{J}_{F}=\mathcal{J}_{B}$, with the LL term, is about twice as large as that of ${ }^{152-158} \mathrm{Sm}$. This dramatic change is consistent with the experiment, although somewhat overshoots experimental changes.

We shall discuss eigenvalues of $H_{B}^{\prime}$ in Eq. (3) obtained by the diagonalization using NPBOS code [34]. We first investigate to what extent $E_{x}\left(2_{1}^{+}\right)$is lowered by the LL term. Figure 3 shows the fraction of this lowering by normalizing it with respect to the $E_{x}\left(2_{1}^{+}\right)$without the LL term, for (a) Sm and (b) U isotopes. This lowering is, as indicated by the arrows in Fig. 3, >30\% for ${ }^{154-158} \mathrm{Sm}$ and $>60 \%$ for ${ }^{232-236} \mathrm{U}$. On the other side, it almost vanishes or is quite small for ${ }^{146-152} \mathrm{Sm}$. Thus, it may not affect the IBM description much, even if one keeps the LL term in all nuclei. We do not take it, because the present derivation does not give a physical basis for the LL term for nuclei without strong deformation.

Figure 4 shows the evolution of low-lying yrast spectra for (a) Sm and the neighboring (b) Gd isotopes as functions of $N$. For both $\mathrm{Sm}$ and $\mathrm{Gd}$ isotopes, the LL term is included for $N \geqslant 90$, but is not included for $N \leqslant 88$, based on the criterion discussed above. The IBM parameters for $\mathrm{Gd}$ isotopes are derived similarly to those used for Sm isotopes. Figures 4(a) and 4(b) indicate that the calculated spectra become more 

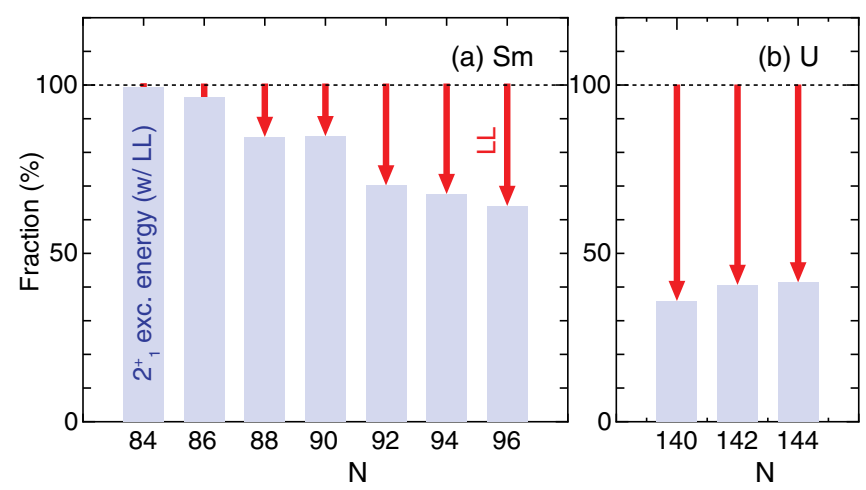

FIG. 3. (Color online) Fraction of $E_{x}\left(2_{1}^{+}\right)$with the LL term, normalized with respect to $E_{x}\left(2_{1}^{+}\right)$without the LL term, for (a) Sm and (b) $\mathrm{U}$ isotopes. Arrows represent the LL matrix elements normalized by $E_{x}\left(2_{1}^{+}\right)($w/o LL).

compressed with $N$ and exhibit a rotational feature for $N \geqslant 90$, which is similar to the experimental trends [35]. One notices a certain deviation at $N=88$, where the Skyrme PES favors stronger deformation and the calculated excitation energies are somewhat too low [12].

Figure 5 shows yrast levels of ${ }^{154} \mathrm{Sm},{ }^{156} \mathrm{Gd},{ }^{230} \mathrm{Th}$, and ${ }^{230} \mathrm{U}$ nuclei as representatives of rotational nuclei. The LL term is included for these nuclei, as they fulfill the criterion. For $^{230} \mathrm{Th}$, the parameters of $H_{B}$ take almost the same values as those
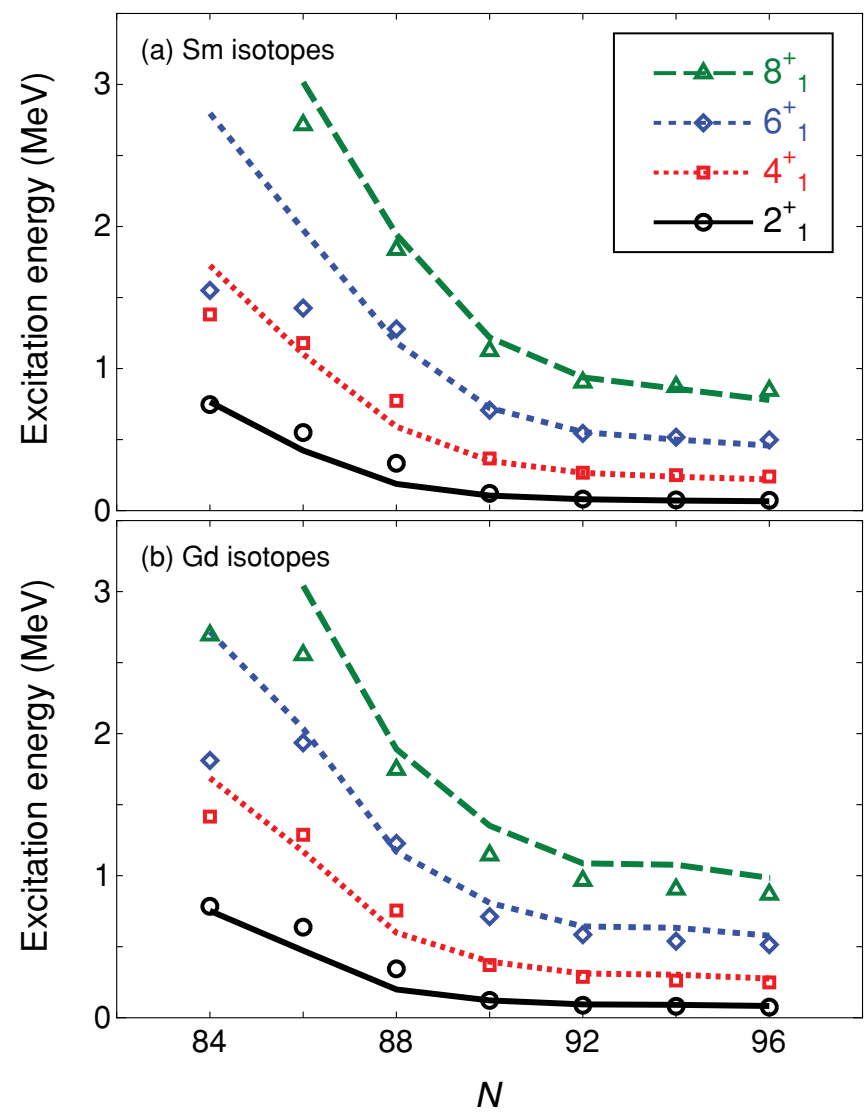

FIG. 4. (Color online) Experimental [35] (symbols) and calculated (curves) yrast spectra for (a) Sm and (b) Gd isotopes.

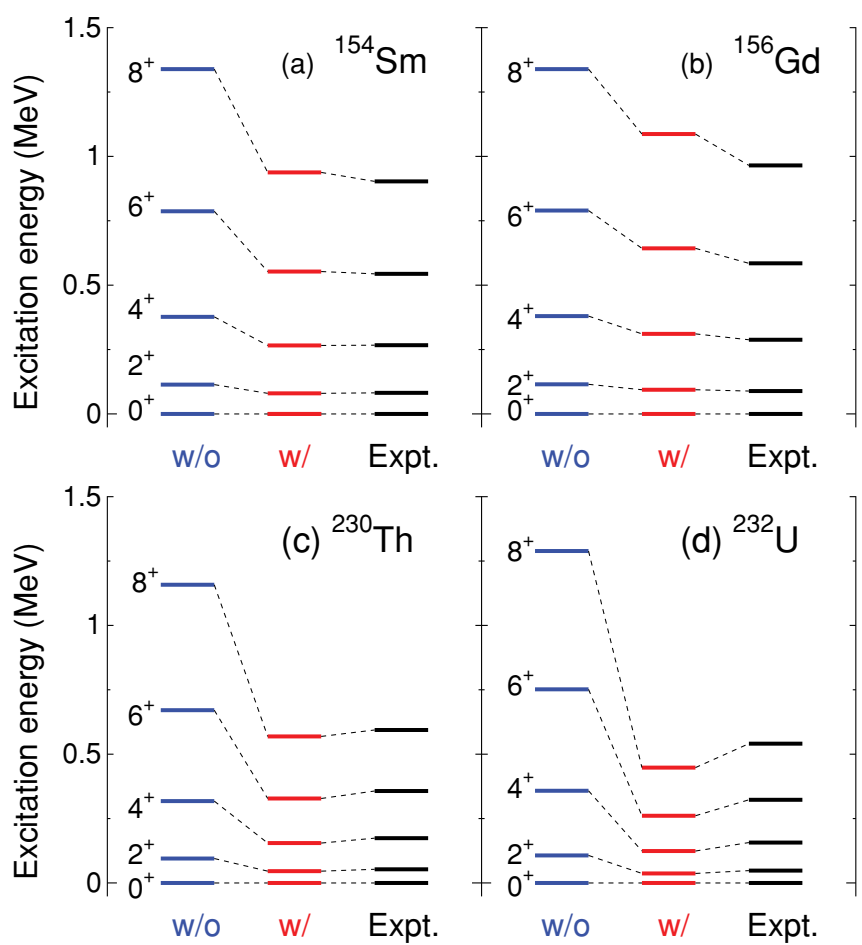

FIG. 5. (Color online) Level schemes of (a) ${ }^{154} \mathrm{Sm}$, (b) ${ }^{156} \mathrm{Gd}$, (c) ${ }^{230} \mathrm{Th}$, and (d) ${ }^{232} \mathrm{U}$ nuclei. Calculated spectra, with $(\mathrm{w} /)$ and without (w/o) the LL term, are compared with experimental [35] spectra.

for the ${ }^{232} \mathrm{U}$ nucleus. A nice overall agreement arises between the theoretical and the experimental [35] spectra, and the contribution of the LL term to it is remarkable. Particularly for ${ }^{154} \mathrm{Sm}$ and ${ }^{230} \mathrm{Th}$, the calculated spectra look nearly identical to the experimental ones.

We now comment on sideband levels. The deviations of $\beta$ bandhead $\left(0_{2}^{+}\right)$and $\gamma$-bandhead $\left(2_{2}^{+}\right)$energies are improved by tens of $\mathrm{keV}$ by the LL term. However, these bandhead energies are still much higher than the experimental ones. Thus, there are still open questions on sideband levels. However, the relative spacing inside the bands is reduced by hundreds of $\mathrm{keV}$, producing certain improvements.

We mention some studies deriving a collective Hamiltonian from a given EDF where the mean-field PES, supplemented with zero-point rotational and vibrational corrections, is treated as an effective potential [36-38]. A generalized kineticenergy term emerges in such approaches. In the present work, we compare the results of Skyrme EDF with the corresponding results of the mapped boson system, at the levels of the PES and the rotational response. The kinetic energies of nucleons are included in both levels, while the rotational kineticlike boson term appears from the latter.

In summary, we have proposed a novel formulation of the IBM for rotational nuclei. The rotation of a strongly deformed, multinucleon system differs in its response to the rotational cranking from its boson image obtained by the mapping method of Ref. [8], where the PES at rest is considered. Significant differences then appear in the moment of inertia between the nucleon and boson systems. We have 
shown that this problem is remedied by introducing the LL term into the IBM Hamiltonian. The effect of the LL term makes an essential contribution to the rotational spectra, solving the longstanding problem of the too-small moment of inertia microscopically. Experimental data are reproduced quite well, without any phenomenological adjustment. The mapping of Ref. [8] appears quite sufficient for vibrational and $\gamma$-unstable nuclei, and the present study makes the IBM description of strongly deformed nuclei sensible theoretically and empirically. Thus, we seem to have obtained a microscopic basis of the IBM in all situations at the lowest order. However, this achievement is partly due to the successful description of the Skyrme mean-field model. The feature discussed in this
Rapid Communication is related to the question of whether or not the IBM can be applied to deformed nuclei [22]. The present work indicates that the rotational response is substantially different between fermions and bosons, but the difference can be incorporated into the IBM in a microscopic way.

The authors acknowledge Dr. A. Gelberg and Dr. D. Vretenar for valuable comments. This work was supported in part by Grants-in-Aid for Scientific Research (A) No. 20244022 and No. 217368, and by the JSPS Core-to-Core program EFES. Authors K.N. and L.G. acknowledge support from the JSPS.
[1] A. Bohr and B. R. Mottelson, Nuclear Structure, Vols. I and II (Benjamin, New York, 1969/1975).

[2] P. Ring and P. Schuck, The Nuclear Many-Body Problem (Springer, Berlin, 1980).

[3] F. Iachello and A. Arima, The Interacting Boson Model (Cambridge University Press, Cambridge, 1987).

[4] A. Frank and P. Van Isacker, Algebraic Methods in Molecular and Nuclear Structure Physics (Wiley, New York, 1994).

[5] T. Otsuka, A. Arima, F. Iachello, and I. Talmi, Phys. Lett. B 76, 139 (1978); T. Otsuka, A. Arima, and F. Iachello, Nucl. Phys. A 309, 1 (1978).

[6] T. Mizusaki and T. Otsuka, Prog. Theor. Phys. Suppl. 125, 97 (1997).

[7] T. Otsuka, Phys. Lett. B 138, 1 (1984); T. Otsuka and N. Yoshinaga, ibid. 168, 1 (1986).

[8] K. Nomura, N. Shimizu, and T. Otsuka, Phys. Rev. Lett. 101, 142501 (2008)

[9] T. H. R. Skyrme, Nucl. Phys. 9, 615 (1959).

[10] D. Vautherin and D. M. Brink, Phys. Rev. C 5, 626 (1972).

[11] M. Bender, P.-H. Heenen, and P.-G. Reinhard, Rev. Mod. Phys. 75, 121 (2003).

[12] K. Nomura, N. Shimizu, and T. Otsuka, Phys. Rev. C 81, 044307 (2010).

[13] T. Otsuka, "Boson Model of Medium-Heavy Nuclei," Ph.D. thesis, University of Tokyo, 1979.

[14] T. Otsuka, Phys. Rev. Lett. 46, 710 (1981).

[15] T. Otsuka, Nucl. Phys. A 368, 244 (1981).

[16] O. Scholten, Phys. Rev. C 28, 1783 (1983).

[17] N. Yoshinaga, A. Arima, and T. Otsuka, Phys. Lett. B 143, 5 (1984).

[18] M. R. Zirnbauer, Nucl. Phys. A 419, 241 (1984).
[19] T. Otsuka and J. N. Ginocchio, Phys. Rev. Lett. 55, 276 (1985).

[20] W. Pannert, P. Ring, and Y. K. Gambhir, Nucl. Phys. A 443, 189 (1985)

[21] T. Otsuka and M. Sugita, Phys. Lett. B 215, 205 (1988).

[22] A. Bohr and B. R. Mottelson, Phys. Scr. 22, 468 (1980).

[23] T. Otsuka, A. Arima, and N. Yoshinaga, Phys. Rev. Lett. 48, 387 (1982).

[24] D. R. Bes, R. A. Broglia, E. Maglione, and A. Vitturi, Phys. Rev. Lett. 48, 1001 (1982).

[25] A. Arima and F. Iachello, Ann. Phys. (NY) 111, 201 (1978).

[26] J. Bartel et al., Nucl. Phys. A 386, 79 (1982).

[27] J. N. Ginocchio and M. W. Kirson, Phys. Rev. Lett. 44, 1744 (1980).

[28] A. E. L. Dieperink, O. Scholten, and F. Iachello, Phys. Rev. Lett. 44, 1747 (1980).

[29] D. R. Inglis, Phys. Rev. 103, 1786 (1956).

[30] S. T. Belyaev, Nucl. Phys. 24, 322 (1961).

[31] L. Guo, J. A. Maruhn, and P.-G. Reinhard, Phys. Rev. C 76, 034317 (2007); L. Guo, J. A. Maruhn, P.-G. Reinhard, and Y. Hashimoto, ibid. 77, 041301(R) (2008).

[32] H. Schaaser and D. M. Brink, Phys. Lett. B 143, 269 (1984).

[33] For instance, R. B. Cakirli, and R. F. Casten, Phys. Rev. Lett. 96, 132501 (2006).

[34] T. Otsuka and N. Yoshida, JAERI-M Report No. 85, 1985.

[35] NuDat 2.5 [http://www.nndc.bnl.gov/nudat2/index.jsp].

[36] P. Bonche et al., Nucl. Phys. A 510, 466 (1990).

[37] J.-P. Delaroche et al., Phys. Rev. C 81, 014303 (2010).

[38] Z. P. Li, T. Nikšić, D. Vretenar, and J. Meng, Phys. Rev. C 81, 034316 (2010). 